\title{
From powerful research platform for industrial EUV photoresist development, to world record resolution by photolithography: EUV interference lithography at the Paul Scherrer Institute
}

\author{
$\underline{\text { Elizabeth Buitrago }}^{1}$, Roberto Fallica ${ }^{1}$, Daniel Fan ${ }^{1}$, Waiz Karim ${ }^{1,2}$, Michaela Vockenhuber ${ }^{1}$, Jeroen \\ A. van Bokhoven ${ }^{2,3}$, and Yasin Ekinci ${ }^{1}$ \\ ${ }^{1}$ Laboratory for Micro-and Nanotechnology, Paul Scherrer Institute, CH-5232 Villigen PSI, \\ Switzerland \\ ${ }^{2}$ Institute for Chemical and Bioengineering, ETH Zurich, Switzerland \\ ${ }^{3}$ Laboratory for Catalysis and Sustainable Chemistry, Paul Scherrer Institute, CH-5232 Villigen PSI, \\ Switzerland
}

\begin{abstract}
Extreme ultraviolet interference lithography (EUV-IL, $\lambda=13.5 \mathrm{~nm}$ ) has been shown to be a powerful technique not only for academic, but also for industrial research and development of EUV materials due to its relative simplicity yet record high-resolution patterning capabilities. With EUV-IL, it is possible to pattern high-resolution periodic images to create highly ordered nanostructures that are difficult or time consuming to pattern by electron beam lithography (EBL) yet interesting for a wide range of applications such as catalysis, electronic and photonic devices, and fundamental materials analysis, among others. Here, we will show state-of the-art research performed using the EUV-IL tool at the Swiss Light Source (SLS) synchrotron facility in the Paul Scherrer Institute (PSI). For example, using a grating period doubling method, a diffraction mask capable of patterning a world record in photolithography of $6 \mathrm{~nm}$ half-pitch (HP), was produced. In addition to the description of the method, we will give a few examples of applications of the technique. Well-ordered arrays of suspended silicon nanowires down to $6.5 \mathrm{~nm}$ linewidths have been fabricated and are to be studied as field effect transistors (FETs) or biosensors, for instance. EUV achromatic Talbot lithography (ATL), another interference scheme that utilizes a single grating, was shown to yield well-defined nanoparticles over large-areas with high uniformity presenting great opportunities in the field of nanocatalysis. EUV-IL is in addition, playing a key role in the future introduction of EUV lithography into high volume manufacturing (HVM) of semiconductor devices for the 7 and $5 \mathrm{~nm}$ logic node (16 nm and $13 \mathrm{~nm} \mathrm{HP}$, respectively) and beyond while the availability of commercial EUV-tools is still very much limited for research.
\end{abstract}

Keywords: EUV Interference Lithography, CAR, photoresist, $11 \mathrm{~nm}$ resolution, $6 \mathrm{~nm}$ resolution

\section{INTRODUCTION}

EUV-IL is a simple lithography technique enabling the production of high-resolution periodic aerial images without the use of expensive high numerical aperture (NA) optics, imaging systems and multilayer reflective photomasks necessary in EUV Microfield exposure tools (METs) or EUV scanners for HVM. ${ }^{1}$ EUV-IL has therefore been extensively used for EUV photoresist testing and development enabling research before industrial exposure tools are available for HVM as will be shown later in this paper. ${ }^{2-4}$ EUV-IL with the use of diffraction-transmission grating masks offers many advantages such as achromaticity, insensitivity to misalignment and infinite depth-of-focus (DOF, insensitive to gap variations between the mask and the substrate). ${ }^{5}$ It is also possible to pattern large areas for cross section analysis at once. Furthermore, the ultimate resolution, i.e. half-pitch (HP), that can be achieved is limited by light diffraction $(\lambda / 4)$ and therefore it is theoretically possible to resolve features down below $4 \mathrm{~nm}$ with EUV-IL lithography at $13.5 \mathrm{~nm}$, a technological feat that still needs to be realized. ${ }^{6}$ At the EUV-IL setup in PSI (XIL-II beamline of SLS) it has been possible to create line/space (L/S) patterns on hydrogen silsesquioxane (HSQ $\sim 15 \mathrm{~nm}$ ) with a resolution down to $6 \mathrm{~nm}$ HP which marks the current world record resolution by photon based lithography as will be shown in the following sections here. ${ }^{7}$

In this paper, we will start by introducing the basic working principle of grating based EUV-IL and continue to describe different examples of applications that benefit from such powerful technique such as patterning of well-ordered

UV and Higher Energy Photonics: From Materials to Applications, edited by Gilles Lérondel, Satoshi Kawata, Yong-Hoon Cho, Proc. of SPIE Vol. 9926, 99260T · — 2016 SPIE · CCC code: 0277-786X/16/\$18 doi: $10.1117 / 12.2238805$ 
structures with ultra-high resolution and the creation of high ordered arrays of suspended silicon nanowires. Furthermore, EUV ATL another powerful interference lithography scheme will be briefly described and its application to nanocatalysis studies will be explored. Finally, the direct measurement of the absorption and Dill's parameters of photoresists at EUV will be discussed.

\section{GRATING BASED EUV-IL}

In grating-based EUV-IL, a mask with transmission-diffraction gratings is illuminated by a spatially coherent beam of EUV light $\lambda$ from an undulator synchrotron source (Swiss Light Source, SLS). Periodic images are then produced by the interference of two or more diffracted coherent beams as schematically shown in Figure 1. The sinusoidal aerial image produced by two beam interference lithography has a period $P$ that is half of the original mask grating period $P_{g}$ when first order $(m=1)$ diffracted beams are used (Equitation 1):

$$
P=\frac{\lambda}{2 \sin \theta_{m}}=\frac{P_{g}}{2 m}
$$

$m$ and $\theta_{m}$ denote the diffraction order and diffraction angle respectively. As it is clear from this equation, the resolution of the generated aerial image can be decreased by the use of higher order diffracted beams $m>1{ }^{8}$ Two linear gratings are used to create lines, while hole/dot patterns can be made in one exposure step by the interference of three or four diffracted beams. ${ }^{9}$ By using multiple beams and controlling their phases, versatile periodic patterns and quasiperiodic periodic patterns can be obtained. ${ }^{10}$

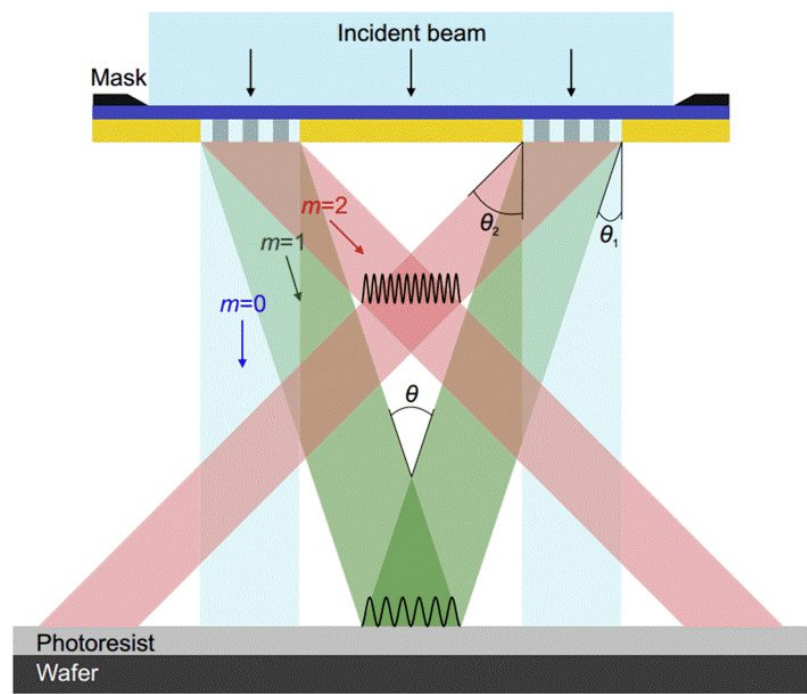

Figure 1. Schematic of EUV-IL set-up where first-order diffraction is used to create an aerial image on resist by interference. Adapted from. ${ }^{11}$

\section{WORLD RECORD RESOLUTION BY PHOTON BASED LITHOGRAPHY BY EUV-IL}

From its first demonstration in the late 1990's by Solak et al., ${ }^{12}$ EUV-IL has broken many photon-based lithography printing resolution records. By the use of monochromatized EUV radiation from an undulator synchrotron source, the authors produced $19 \mathrm{~nm}(\mathrm{~L} / \mathrm{S})$ fringe patterns printed on (100 nm thick) polymethyl methacrylate (PMMA) for the first time with a photon-based lithography technique. ${ }^{12}$

The printing quality and ultimate resolution that can be achieved by EUV-IL can be optimized by focusing in three key areas: photoresist chemistry and processing, stability of the EUV source and the stage, and fabrication of high-resolution diffraction masks with good quality and diffraction efficiency (dependent on the design and grating material). In 
particular, $\mathrm{SiO}_{\mathrm{x}}$ diffraction gratings directly written on silicon nitride $\left(\mathrm{Si}_{3} \mathrm{~N}_{4}\right)$ membranes $(<100 \mathrm{~nm}$ thick $)$ by electron beam lithography (EBL, from HSQ) have been shown to be sufficient to achieve resolutions down to $10 \mathrm{~nm} \mathrm{HP}$ on wafer. ${ }^{3,13}$ Below $10 \mathrm{~nm}$ HP however, $\mathrm{SiO}_{\mathrm{x}}$ drastically reduces in EUV diffraction efficiency $(<<1 \%)$ depending on the geometry (pitch, material thickness, duty cycle: the ratio between linewidth and pitch) and optical properties of the grating. ${ }^{11,14}$

Record high, sub-10 nm resolution dense patterning down to $7 \mathrm{~nm}$ HP has nonetheless been accomplished by EUV-IL at PSI as can be seen in Figure 2. This was accomplished by the use of novel Sn-oxide (Figure 2a, b) and Hf-oxide (Figure 2c) based resists (Inpria Corp). ${ }^{6}, 15$ These resist materials can be easily spin-coated and directly patterned when exposed to an electron or photon beam so that no pattern transfer is required. Using these negative-tone, metal-oxide based resists from Inpria Corp., enable the direct fabrication of gratings with high diffraction efficiencies (improved by at least a factor of two in comparison to when $\mathrm{SiO}_{2}$ gratings are used) necessary for sub-10 nm HP $\left(P_{g} \leq 40 \mathrm{~nm}\right)$ resolution printing. The diffraction efficiency of $\mathrm{HfO}_{\mathrm{x}}$ and $\mathrm{SnO}_{\mathrm{x}}$ gratings is in fact theoretically sufficient to pattern images with a resolution down to $5 \mathrm{~nm} \mathrm{HP}(\sim 3 \%)$, however this still needs to be realized experimentally., ${ }^{6}$
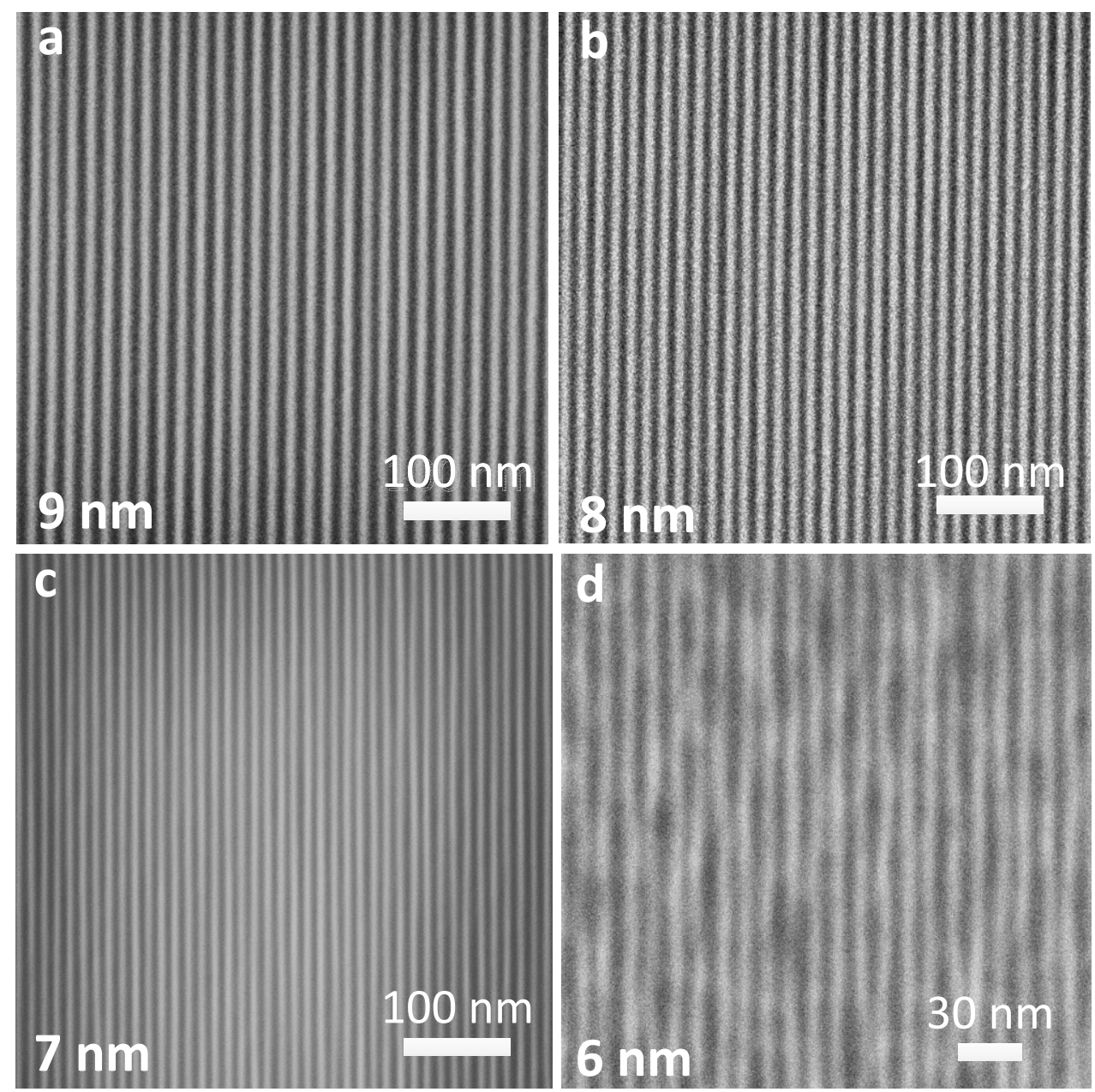

Figure 2. SEM micrographs of L/S patterns with $9 \mathrm{~nm}-6 \mathrm{~nm}$ HP resolution obtained by EUV-IL on HSQ by the use of a, b) $\left.\mathrm{SnO}_{\mathrm{x}}{ }^{13}, \mathrm{c}\right) \mathrm{HfO}_{\mathrm{x}}{ }^{6}$ gratings directly patterned by EBL and, d) Ir gratings fabricated by a pitch doubling method. ${ }^{7}$

Patterning densely packed features in the $\mathrm{nm}$ range by EBL is still nonetheless quite challenging due to electron proximity effects. $^{6-7,13}$ The efficient fabrication of high resolution gratings employing diffraction efficient materials for sub-10 $\mathrm{nm}$ patterning is therefore still critical. Pattern transfer by reactive ion etching (RIE) into high diffraction efficiency materials such as Ir or Ru can be particularly difficult and clever fabrication processes need to be developed. 
In an alternative fabrication method, $\mathrm{SiO}_{\mathrm{x}}$ gratings are written first at a relaxed pitch by EBL followed by atomic layer deposition (ALD) of Ir as shown in Figure 3. Then, by using a directional etching process such as ion milling, iridium at the top and bottom of trench is etched leaving the materials only on the sides of the $\mathrm{SiO}_{\mathrm{x}}$ grating lines. In this manner, a Ir grating with double the pitch of the original $\mathrm{SiO}_{\mathrm{x}}$ grating and high diffraction efficiency can be produced. Using this advanced pitch doubling method, a record resolution of $6 \mathrm{~nm} \mathrm{HP}$ was achieved, Figure $2 \mathrm{~d}^{7} \mathrm{The}^{\mathrm{SiO}} \mathrm{x}_{\mathrm{x}}$ can then be furthermore removed in a buffered HF bath (BHF, not performed here). Although the resulting structures are partially collapsed and exhibit high line-edge roughness, the lines are resolved. The patterning quality at this resolution is moreover far from any practical use at the moment. Still, this is an impressive demonstration that advances state-of-theart research and this mask making approach is very promising, pushing the resolution limits of the technique.
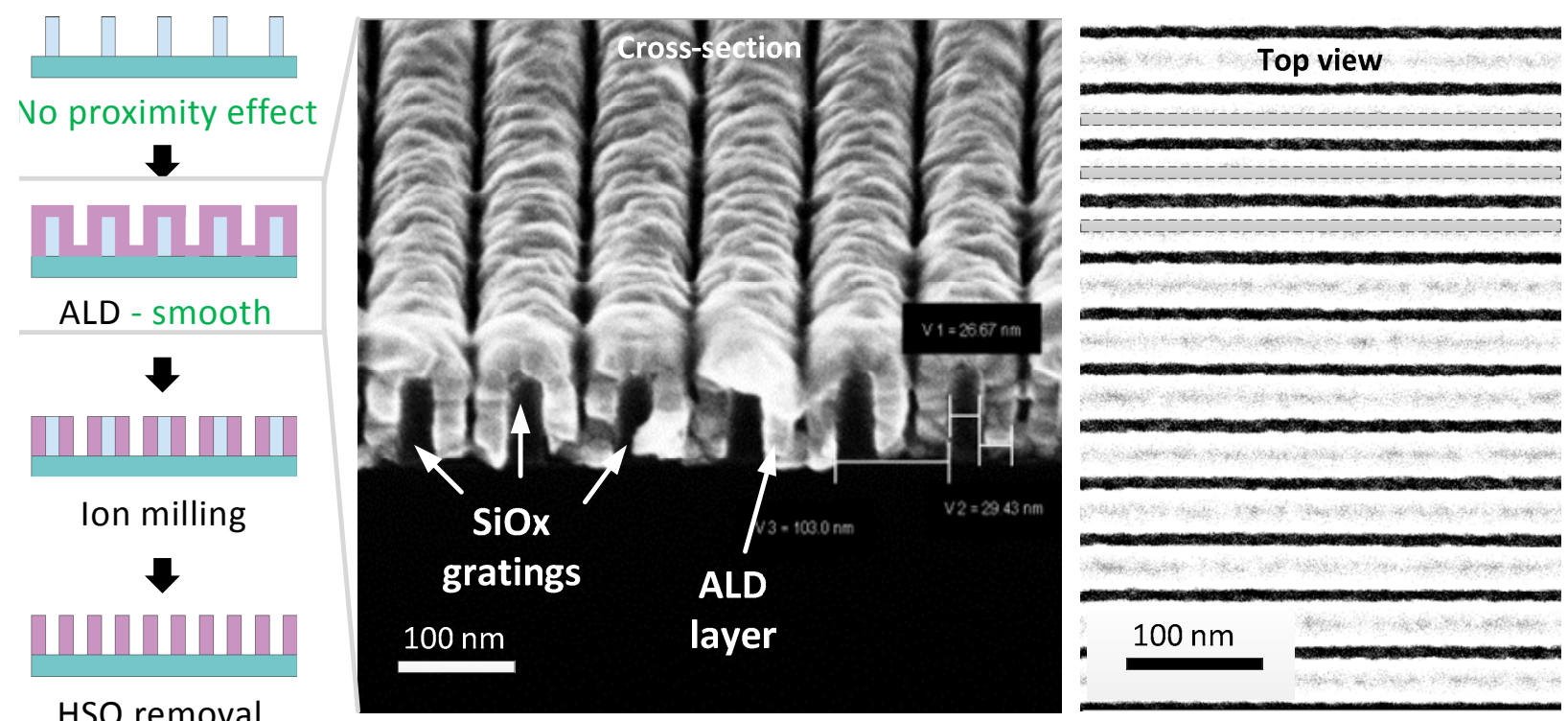

Figure 3a) Pitch doubling fabrication process steps: $\mathrm{SiO}_{\mathrm{x}}$ gratings with a relaxed period fabricated by EBL, conformal Ir deposition around the $\mathrm{SiO}_{\mathrm{x}}$ gratings by $\mathrm{ALD}$, subsequent ion-milling and $\mathrm{SiO}_{\mathrm{x}}$ removal with $\mathrm{BHF}$. SEM b) cross-section and c) top-view of fabricated structure after ALD of Ir with pitch duplicate highlighted in c. ${ }^{7}$

As mention above, in addition to two-beam interference leading to line/space patterns, by increasing the number of interfering beams, periodic patterns such as dots, holes, kagome lattices, honeycombs, and quasicrystals can be fabricated. ${ }^{10,16}$ By increasing the number of interfering beams to infinity, i.e. a ring grating, a Bessel beam can be produced, which has a long, propagation invariant central core that is self-healing and non-diffracting, allowing serial lithography over curved surfaces and topographical features with changes in height. We have demonstrated Bessel beams with a depth-of-focus of up to $1 \mathrm{~mm}$ with a $220 \mathrm{~nm}$ diameter spot as well as Bessel beams down to $30 \mathrm{~nm}$ spot size as can be seen in Figure 4. 


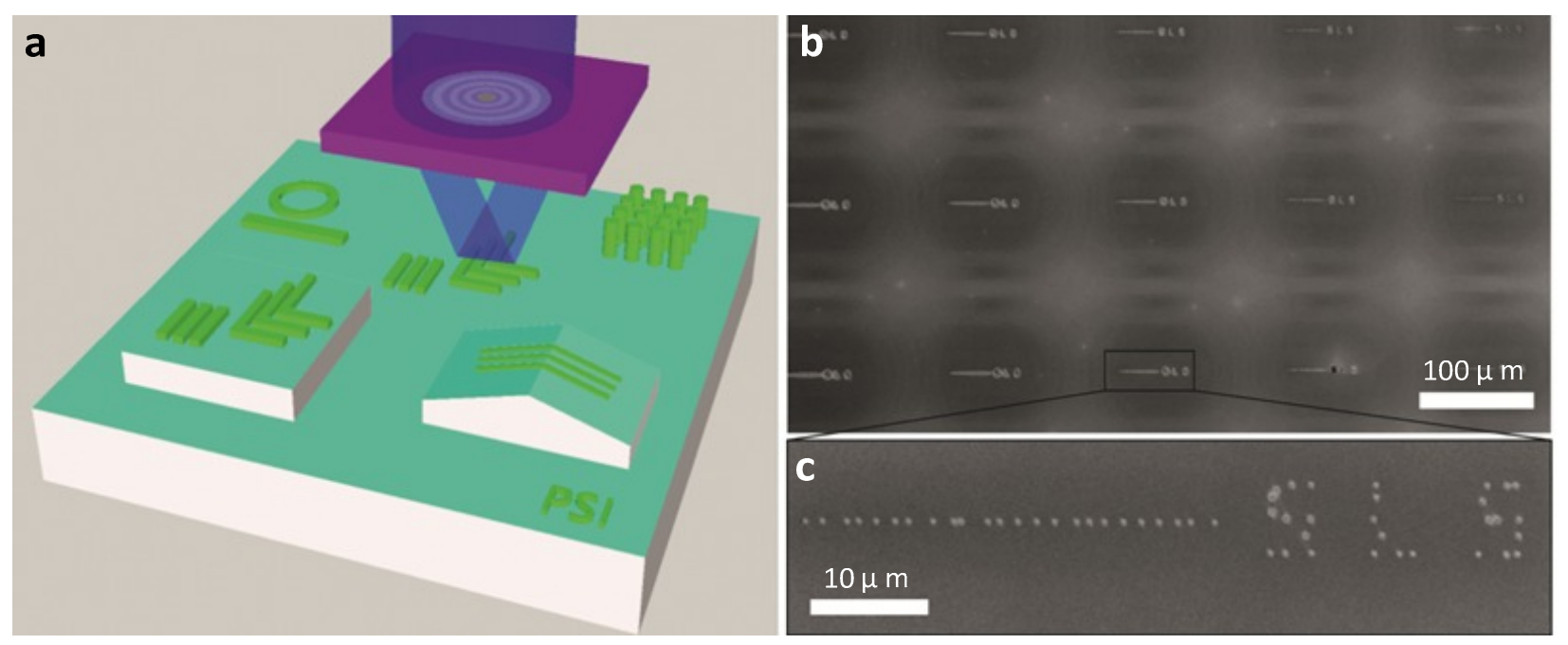

Figure 4a) Schematic of serial lithography over arbitrary topographical features using a Bessel beam of EUV light produced via a ring diffraction grating. b, c) Structures written using a $230 \mathrm{~nm}$ diameter Bessel beam of EUV light with $1 \mathrm{~mm}$ core length. Top scale bar $100 \mathrm{um}$, bottom scale bar $10 \mathrm{um}$.

\section{POWERFUL INDUSTRIAL TOOL FOR PHOTORESIST TESTING AND DEVELOPMENT}

Lithographic performance has been driven by the constant need of device size miniaturization and productivity in the semiconductor industry (Moore's Law). EUVL is now predicted to be introduced in HVM at the $7 \mathrm{~nm}$ node at multiple lithography steps according to ASML's (September 2015) NXE extension roadmap. ${ }^{17}$ With EUVL now finally in the near future, the successful development of high performance EUV materials to resolve features down to $16 \mathrm{~nm}$, $13 \mathrm{~nm} \mathrm{HP}$ and beyond is ever more important. As EUV exposure tools are not yet easily accessible for research, EUV-IL has been shown to be a valuable technique for photoresist research testing before the exposure tools become available for HVM. At PSI we help industry develop and evaluate the performance and ultimate resolution limits of different photoresists for future technology nodes. In the last years we have evaluated hundreds of different state of-the-art EUV materials from different vendors all around the world. Among these, we have identified several promising CAR candidates for future testing that simultaneously meet sensitivity, LWR and exposure latitude (EL) high performance requirements with the aim of resolving (L/S) features for the 7 and $5 \mathrm{~nm}$ logic node (16 nm and $13 \mathrm{~nm} \mathrm{HP}$, respectively) for HVM. ${ }^{2,18}$ The critical dimension (CD) and LWR vs. dose values for 5 different, best highly performing CARs with $\mathrm{BE}<30 \mathrm{~mJ} / \mathrm{cm}^{2}, \mathrm{LWR}<6.5 \mathrm{~nm}(\mathrm{LER}<4.6 \mathrm{~nm})$ and $\mathrm{EL}>15 \%$ at $16 \mathrm{~nm} \mathrm{HP}$ are plotted in Figure 5. The LWR, BE and EL threshold values used here to pick the best resist candidates are arbitrary values not set by industry. We have therefore identified highly competitive CAR candidates necessary for the $7 \mathrm{~nm}$ node and the successful introduction of EUVL into HVM. SEM images at $24.8 \mathrm{~mJ} / \mathrm{cm}^{2}, 30 \mathrm{~mJ} / \mathrm{cm}^{2}$ and $47.2 \mathrm{~mJ} / \mathrm{cm}^{2}$ for UL1R6 have been included in this figure to illustrate how pinching serves to increase the LWR values as the dose increases. 

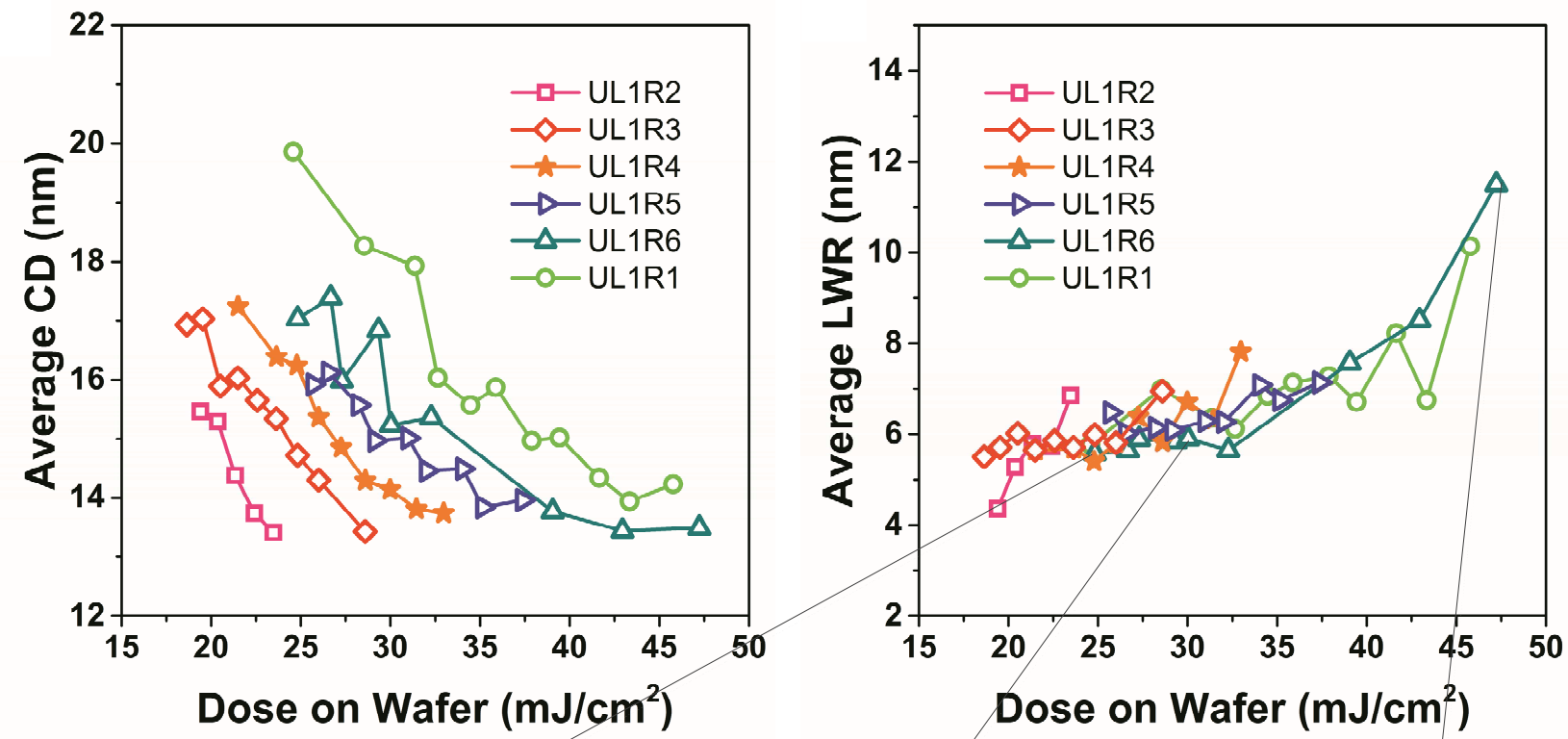

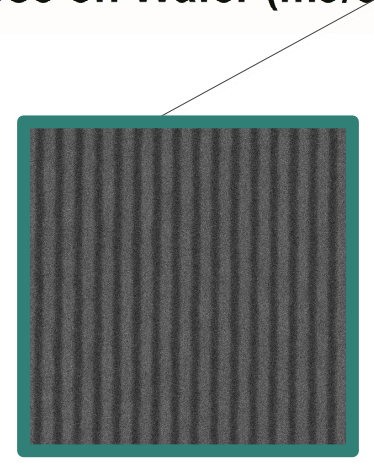

$24.8 \mathrm{~mJ} / \mathrm{cm} 2$

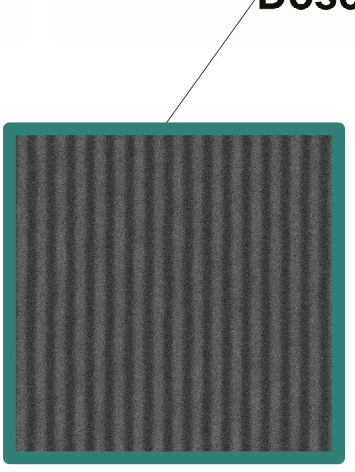

$30 \mathrm{~mJ} / \mathrm{cm} 2$

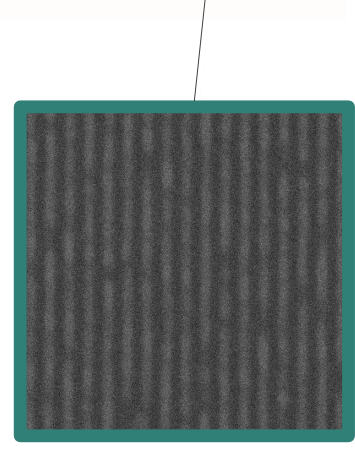

$47.2 \mathrm{~mJ} / \mathrm{cm} 2$

Figure 5. Average CD vs. dose (a) and average LWR vs. dose (b) graphs for the highest performing resists (R2-R6) tested (BE values $<30 \mathrm{~mJ} / \mathrm{cm}^{2}, \mathrm{LWR}<6.5 \mathrm{~nm}$ and EL $>15 \%$, these are arbitrary threshold values not industry targets) at $16 \mathrm{~nm}$ HP resolution from. ${ }^{2,18}$ UL1R1 is the reference resist. All of the resists presented in these graphs are positive tone. SEM images at $24.8 \mathrm{~mJ} / \mathrm{cm}^{2}, 30 \mathrm{~mJ} / \mathrm{cm}^{2}$ and $47.2 \mathrm{~mJ} / \mathrm{cm}^{2}$ for UL1R6 have been included to illustrate how pinching serves to increase the LWR values as the dose increases.

Various CARs that were tested last year were found to be well resolved down to $13 \mathrm{~nm}$ HP. At $13 \mathrm{~nm}$ HP resolution and below nonetheless, pattern collapse, bridging and pinching still limits the EL. The high performance arbitrary threshold values (not set by industry) requirements that need to be met simultaneously at such high resolution have been slightly relaxed and three high performing candidates have been determined. The best CAR candidates at $13 \mathrm{~nm}$ resolution simultaneously show $\mathrm{BE}<50 \mathrm{~mJ} / \mathrm{cm}^{2}, \mathrm{LWR}<8 \mathrm{~nm}(\mathrm{LER}<5.6 \mathrm{~nm})$ and $\mathrm{EL}>3 \%$. We have furthermore found three several CAR resists that are well resolved down to $12 \mathrm{~nm}$ HP and can be resolved down to $11 \mathrm{~nm}$ HP with minimal pattern collapse and bridging, as can be seen in Figure 6 for resist UL1R1. Though a remarkable feat for CARs, it is clear from these results that there is an impending need for alternative resist solutions at $13 \mathrm{~nm}$ resolution and below. 


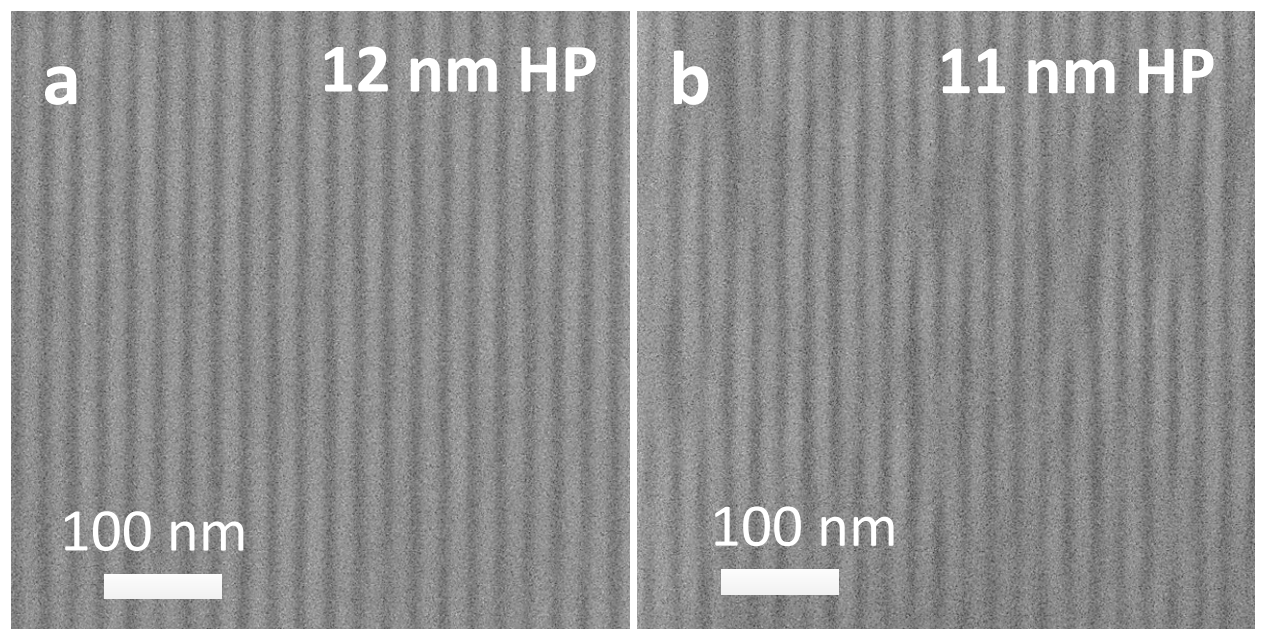

Figure 6. SEM (L/S) images showing one of the highest performing resists at 12 (a) and (b) $11 \mathrm{~nm}$ HP resolution. Minimal pattern collapse and bridging are observed. Adapted from. ${ }^{18}$

\section{FUNCTIONAL WELL-ORDERED NANO-ARRAYS PRODUCED BY EUV-IL}

High-resolution patterns produced by EUV-IL can be subsequently transferred into functional materials. $12 \mathrm{~nm}$ lines, for example, have been previously transferred into silicon-on-insulator (SOI) substrates using reactive ion etching (RIE) in an inductively coupled plasma (ICP) tool to produce silicon nanowires (SiNWs, Figure 7a). This method allows access to NW sizes typically achieved only by growth methods while providing geometric order and strict size tolerances to enable device integration. The etched silicon nanowires can be released from the buried oxide (BOX) in a BHF bath to form field effect transistor (FET) channels to be gated-all-around. The nanowires can be furthermore thinned down by the use of Piranha $\left(\mathrm{H}_{2} \mathrm{O}_{2}: \mathrm{H}_{2} \mathrm{SO} 4,7: 1\right)$ and BHF solutions in consecutive cycles to create and successively remove a chemical oxide around the nanostructures. Using this technique, suspended silicon nanowires with line-widths down to $6.5 \mathrm{~nm}$ have been produced as can be seen in Figure $7 \mathrm{~b}$.
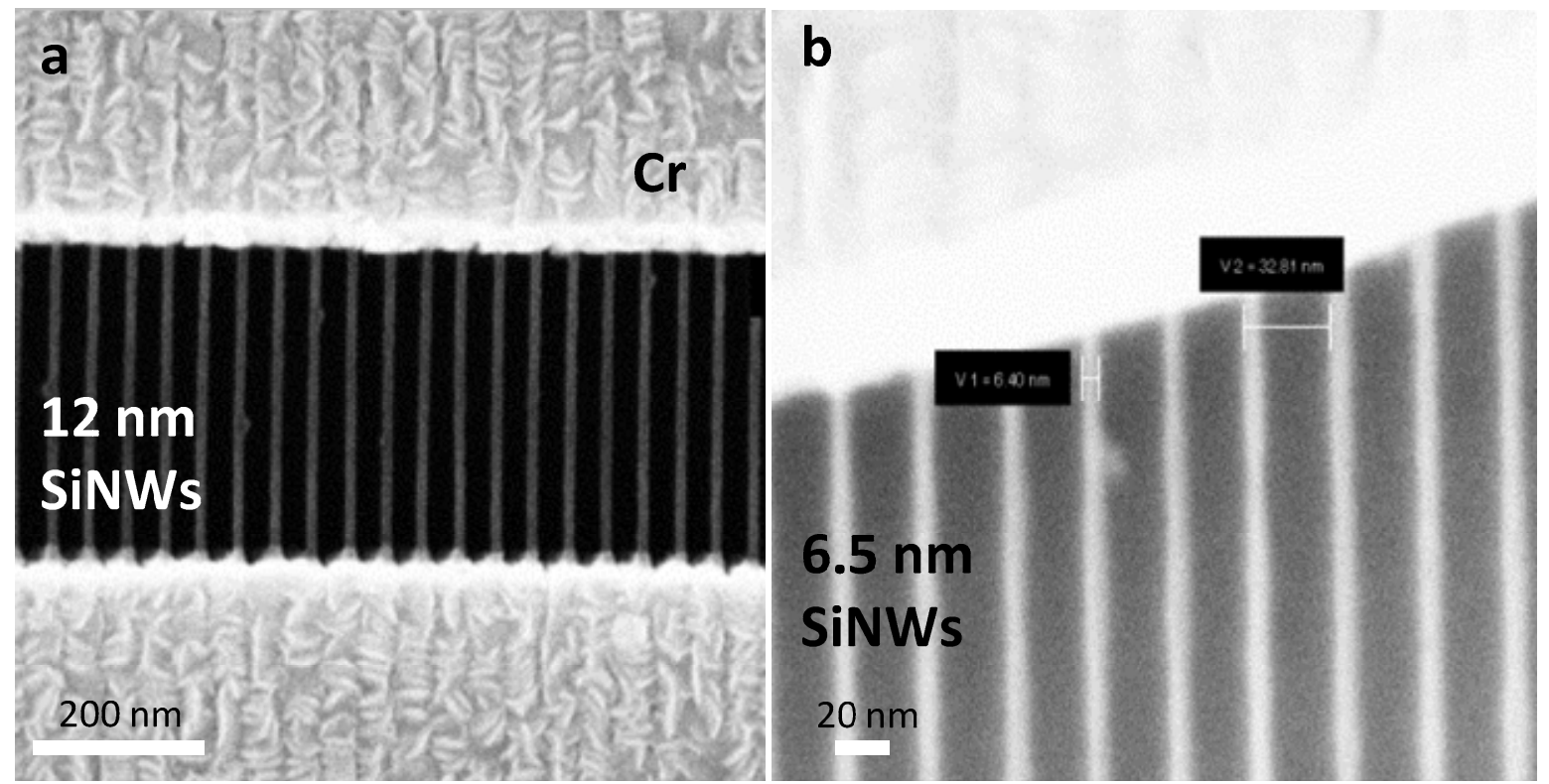

Figure 7. Top-view SEM micrographs of SiNWs with $12 \mathrm{~nm}$ and $6.5 \mathrm{~nm}$ line-widths (a) directly transferred by RIE from patterns created by EUV-IL and (b) thinned down by consecutive Piranha and BHF cycles. 
Raman spectroscopy was additionally employed to probe the thermal and strain properties of the nanowires. Preliminary results show thermal conductivity of silicon nanowires decreased to $1 / 100^{\text {th }}$ of the bulk silicon value. Line-width had a negligible effect on thermal conductivity amongst nanowires, while line-edge-roughness had a large effect of decreasing the thermal conductivity almost 100 -fold close to the amorphous silicon limit (from $\sim 150 \mathrm{~W} / \mathrm{m} \cdot \mathrm{K}$ down to $\sim 2 \mathrm{~W} / \mathrm{m} \cdot \mathrm{K}$ ), which can be attributed to phonon boundary scattering. Low thermal conductivity combined with good electrical conductivity is necessary for efficient thermoelectric harvesting. Silicon nanowires are also standout candidates for future transistor devices as they allow all-around-gating to improve electrostatic control of the channel. However, their resistivity is known to increase as the line-width decreases, which can be mitigated by applying strain to improve carrier mobility and thus decrease resistivity. We show that elastic strain varies with the processing method, opening the door to controllable process induced elastic strain for mobility enhancement.

\section{LARGE-AREA NANOPARTICLE ARRAYS AS MODEL SYSTEMS FOR CATALYSIS USING EUV-ATL}

Another application of EUV-IL owing to its high-resolution and relatively high throughput is the development of model systems with precise nanoparticle size and position over large areas. Metal nanoparticles are important in many catalytic applications. Conventional chemical synthesis techniques result in lack of order, precise particle position and size control necessary for fundamental catalysis analysis. Synthesis of model systems in a controlled manner resulting in welldefined shapes, size and position of catalytic particles as well as their study at the single particle level is necessary to gain deeper insight into chemical mechanisms in heterogeneous systems. For this reason, novel catalytic model surfaces have been patterned using EUV-IL ${ }^{19}$ and $\mathrm{EBL}^{20}$ at PSI.

Understanding size effects in nanoparticles is of great interest in catalysis and the effect of nanoparticle confinement on the catalytically active sites was addressed using lithographically prepared model system. A systematic study of the sizedependent behavior of iron/iron oxide nanoparticles was performed at the X-ray photoemission electron microscope (XPEEM) also located at the SLS in PSI enabling in-situ spectromicroscopy on individual iron particles prepared with EBL. ${ }^{20}$ This combination opens up a new dimension in research to solve fundamental questions in catalysis by visualizing model systems representing more complex catalytic phenomenon. Classical tools to characterize catalytic particles such X-ray photoemission spectroscopy require dense packing of uniform particle sizes over a large-area to yield good single-to-noise ratio. A limitation of EBL prepared model systems is the low-throughput, which limits patterning over large-area making such model systems incompatible with most common analytical instruments. For this reason, EUV-IL capable of patterning high-resolution periodic structures with relatively high throughput has been considered for this application.

Four-beam EUV-IL with a transmission gratings in nickel yields uniformity in size, ${ }^{19}$ but results in first order noninterfering diffraction patterns around the main field limiting true large area patterning and effective stitching. Using a similar fabrication strategy to produce multiple-beam transmission-diffraction grating masks, achromatic Talbot lithography (ATL) masks have been produced. Such masks were used for single-shot patterning over $485 \times 485 \mu \mathrm{m}^{2}$ area, which was extended for ultra-large area patterning over more than $1 \times 1 \mathrm{~cm}^{2}$ area using a step-and-repeat exposure scheme (Figure 8a). ${ }^{19}$ Dot arrays with sizes down to $15 \mathrm{~nm}$ in diameter and $100 \mathrm{~nm}$ pitch and have been achieved employing this strategy (Figure 8b). ${ }^{19,21}$ ATL is in fact a very efficient way to pattern large areas quickly, and uses the spectral distribution of the EUV light to its advantage. With ATL, at a certain distance from the transmission mask, called the achromatic distance, smearing and overlap of the self-images of the mask gives rise to a stationary intensity pattern. Filtering of the high spatial frequencies makes EUV exposure results immune to surface defects and edge roughness of the mask. As can be seen in Figure 8a, only a small non-interfering area between each single-shot exposure (stitching of multiple fields) exists. This results in nanoparticle arrays patterned over large areas for catalysis studies. Such high-resolution patterns generated using EUV-ATL, in addition, have potential in fields that require better understanding of the relationship between optical, electronic, and magnetic properties of nanoparticles and their structure. 


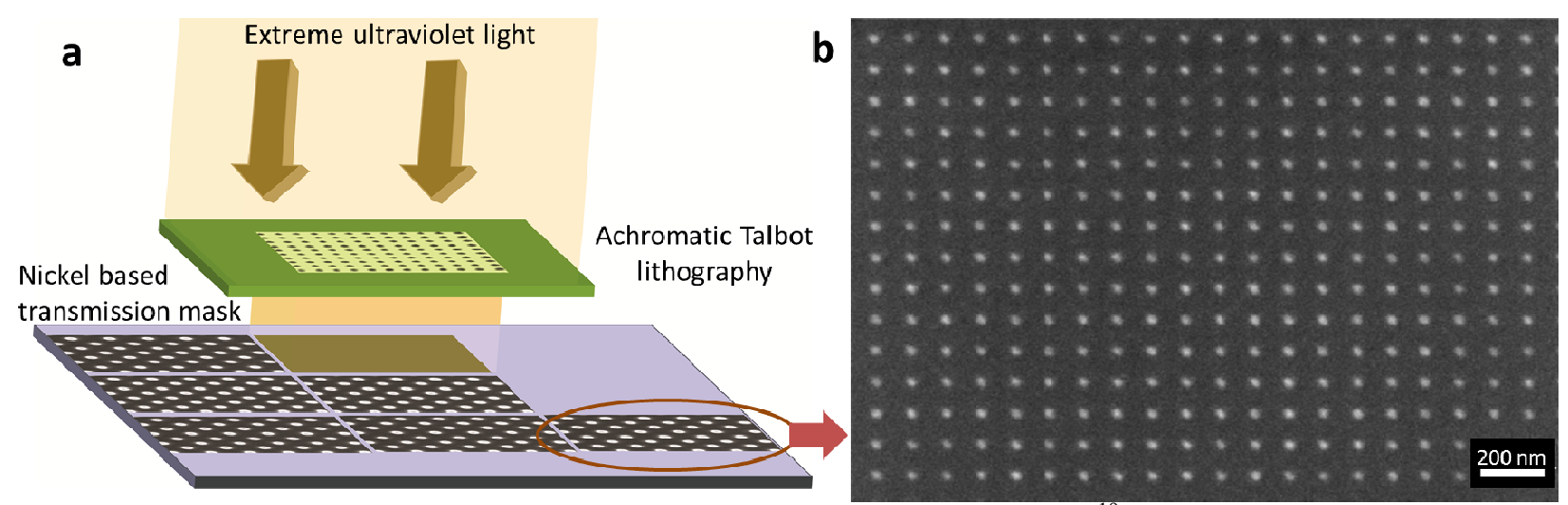

Figure 8. (a) Scheme of EUV-ATL for large area patterning of nanodots, adapted from ${ }^{19}$, employing step-and-repeat exposure resulting in (b) $15 \mathrm{~nm}$ dots with $100 \mathrm{~nm}$ period (SEM image).

\section{NOVEL METHOD FOR PHOTORESIST ABSORPTION MEASUREMENTS AT EUV}

Another method that we have recently developed is the direct measurement of the absorption and Dill's parameters of photoresists at EUV. ${ }^{22-23}$ The absorption coefficient defines the proportion of photons that interact with the resist material while passing through it. Optimum photolithography absorption results from a balance between efficiently harvesting incoming photons (high absorption) and the need to maintain constant intensity throughout the film thickness (low absorption). The absorption coefficient is a key parameter in the simulation and modeling of the dose response of a photoresist and it is especially relevant in EUV lithography. This is because the photon density (\# photons $/ \mathrm{cm}^{3}$ ) for the same dose $\left(\mathrm{mJ} / \mathrm{cm}^{2}\right)$ is significantly smaller than it was in pre-EUV technologies. In fact, the photon density for $193 \mathrm{~nm}$ ArF excimer laser lithography is about 14 times higher in comparison to EUV at the same dose. ${ }^{24-25}$ As a result, both the quantification of the effective dose as a function of resist thickness/depth and the estimation of photon shot-noise depend of the reliable measurement of the absorption coefficient. However, not many studies on this topic have been conducted, owing to the lack of bright EUV sources and accurately being able to measure sub-micrometer film thickness.

In the experimental setup at PSI, the intensity $I$ of EUV light passing through a thin film of photoresist coated on a semitransparent silicon nitride membrane is measured in transmission mode as can be seen in Figure 9. A photodiode, located behind the membrane is used to measure the amount of absorbed light. Because the intensity of the beam is comparable to that of a conventional EUV exposure, we also measure the variation of absorption during the EUV exposure. These dynamic absorption coefficients are known as the Dill parameters and are of technological importance for simulation and modeling. The transmittance $T_{x}$ is given by the ratio of the measured photocurrent $I$ and the reference photocurrent $I_{0}$ (reference measured through blank silicon nitride membrane) and relates to the resist thickness $d$ and linear absorption confident as can be seen in Equation 2.

$$
T_{x}=e^{-\alpha d}
$$

To extract $\alpha\left[\mu \mathrm{m}^{-1}\right]$, the thickness of the resist layer needs to be known accurately which is not straightforward when coating a flexible membrane. Here we used a spectroscopic ellipsometer (Woollam M-2000) with focusing probe of $30 \mu \mathrm{m}$ resolution to map the coated thickness and obtain an accurate measurement of these nanometer thick films. 

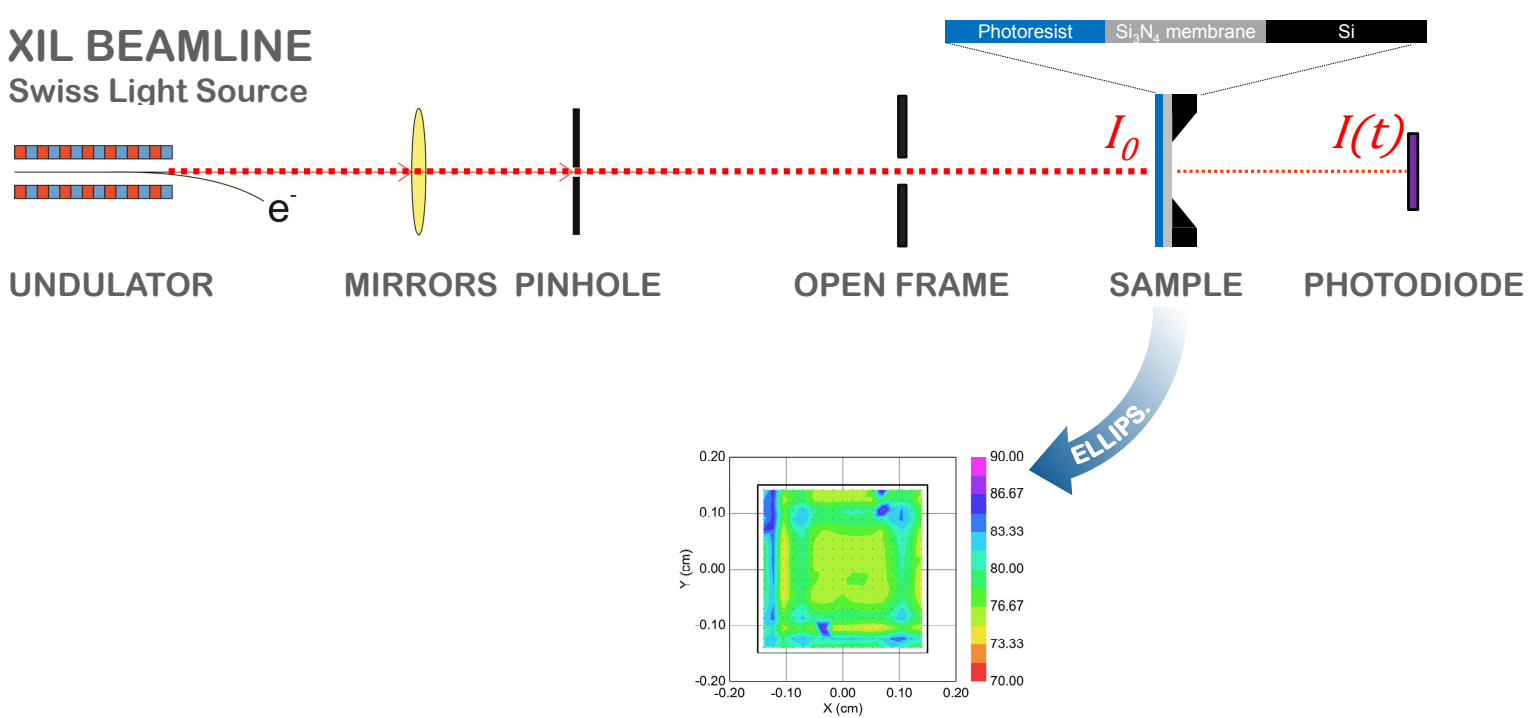

Figure 9. Schematic of experimental set-up for absorption measurements, adapted from. ${ }^{23}$

Most of the EUV photoresists tested here are based on organic polymer backbones with absorption coefficients ranging from 3 to $5 \mu \mathrm{m}^{-1}$ as they are composed mostly of carbon and oxygen. Moreover, we had the opportunity to measure novel EUV photoresist which incorporate heavier elements (meant to improve the absorption). To validate our experimentally determined adsorption coefficients we calculated the theoretical values of the same resists as can be seen in Figure 10. It is clear from this figure that our experimental values match very well with the theoretically calculated values. Furthermore, our results for PMMA and HSQ in particular are in good agreement with previous experimental studies. We determined experimentally a remarkable increase in $\alpha$ (up to $20 \mu \mathrm{m}^{-1}$ ) for the metal-containing formulations. This methodology provides unprecedented accuracy in the measurement of the absorption coefficient at EUV. ${ }^{23}$

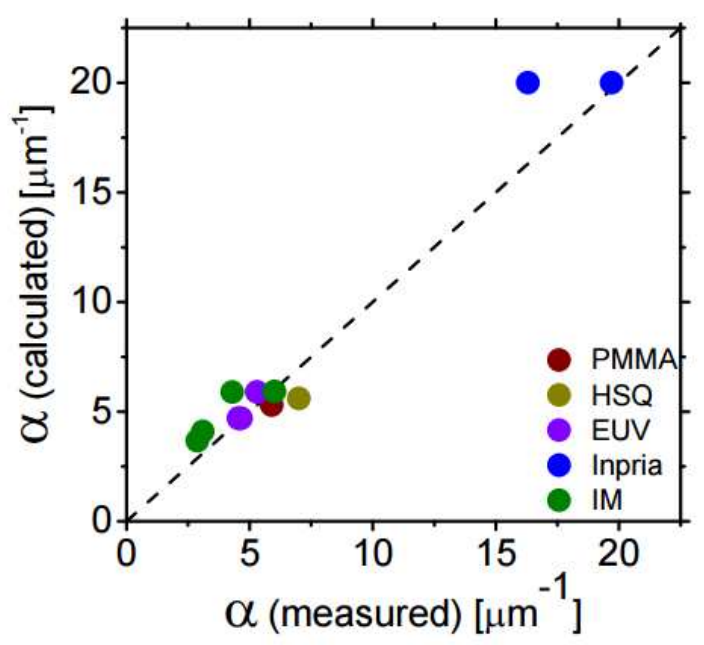

Figure 10. Comparison between experimental and theoretical absorption coefficients for different resist, adapted from. ${ }^{23}$

\section{CONCLUSIONS}

EUV-IL is a simple yet powerful technique that has been used not only for the characterization and development of novel EUV materials but also for the fabrication of high-resolution periodic structures. Patterning down to $6 \mathrm{~nm}$ HP has been accomplished by EUV-IL. This is the smallest feature-size that has been patterned by photolithography up to date. Furthermore, several promising EUV material candidates that simultaneously meet sensitivity, LWR and EL high 
performance requirements with the aim of resolving line space (L/S) features for the 7 and $5 \mathrm{~nm}$ logic node $(16 \mathrm{~nm}$ and $13 \mathrm{~nm} \mathrm{HP}$, respectively) have been identified for future investigation. Also, for the first time CARs were found to be well resolved down to $12 \mathrm{~nm}$ and $11 \mathrm{~nm} \mathrm{HP}$ with minimal pattern collapse and bridging, a remarkable feature for CARs. Uniform nanoparticles over large areas have been achieved using EUV-ATL with step-and-repeat exposure strategy, which has wide range of applications in areas such as catalysis. At the XIL-II beamline we have furthermore developed a new methodology for the accurate measurement of photoresist absorption coefficients necessary for the continuous development of EUV materials for future technology node HVM. We have furthermore EUV-IL to produce silicon nanowires with quantum dimensions to study the materials properties of these versatile structures, and their applications to future devices such as thermoelectric harvesters and gate-all-around transistors with mobility strain enhancement. These are just some of the examples of the state-of-the-art research that goes on at the EUV-IL beamline at PSI.

\section{REFERENCES:}

[1] Naulleau, P. P.; Anderson, C. N.; Horne, S. F., Extreme ultraviolet interference lithography with incoherent light. In Emerging Lithographic Technologies XI, Lercel, M. J., Ed. Bellingham, 2007; Vol. 6517, pp T5172-T5172.

[2] Buitrago, E.; Nagahara, S.; Yildirim, O., et al. "Sensitivity enhancement of chemically amplified resists and performance study using EUV interference lithography," Proc. SPIE Advanced Lithography, pp 97760Z-97760Z-15, (2016).

[3] Buitrago, E.; Yildirim, O.; Fallica, R., et al. "The road towards single digit nanometer resolution patterning in mass production: State-of-the-art EUV resists platforms compared," Proc. EUVL Symposium, Maastricht, The Neatherlands, (2015).

[4] Buitrago, E.; Yildirim, O.; Verspaget, C., et al. "Evaluation of EUV Resist Performance Using Interference Lithography," Proc. SPIE Advanced Lithography, pp 94221S-94221S-13, (2015).

[5] Auzelyte, V.; Dais, C.; Farquet, P., et al., "Extreme Ultraviolet Interference Lithography at the Paul Scherrer Institut," Journal of Micro/Nanolithography, MEMS, and MOEMS(8) 2, 021204-021204-10, (2009).

[6] Mojarad, N.; Hojeij, M.; Wang, L., et al., "Single-Digit-Resolution Nanopatterning with Extreme Ultraviolet Light for the $2.5 \mathrm{~nm}$ technology Node and Beyond," Nanoscale(7) 9, 4031-4037, (2015).

[7] Fan, D.; Ekinci, Y. "Photolithography reaches $6 \mathrm{~nm}$ half-pitch using EUV light," Proc. SPIE Advanced Lithography, San Jose, pp 97761V-97761V-11, (2016).

[8] Päivänranta, B.; Langner, A.; Kirk, E., et al., "Sub-10 nm Patterning Using EUV Unterference Lithography," Nanotechnology(22) 37, 375302, (2011).

[9] Terhalle, B.; Langner, A.; Päivänranta, B., et al. "Advanced holographic methods in extreme ultraviolet interference lithography," Proc. pp 81020V-81020V-7, (2011).

[10] Langner, A.; Päivänranta, B.; Terhalle, B., et al., "Fabrication of Quasiperiodic Nanostructures with EUV interference Lithography," Nanotechnology(23) 10, 105303, (2012).

[11] Mojarad, N.; Gobrecht, J.; Ekinci, Y., "Interference Lithography at EUV and Soft X-ray Wavelengths: Principles, Methods, and Applications," Microelectronic Engineering(143), 55-63, (2015).

[12] Solak, H. H.; He, D.; Li, W., et al., "Exposure of 38 nm Period Grating Patterns with Extreme Ultraviolet Interferometric Lithography," Applied Physics Letters(75) 15, 2328-2330, (1999).

[13] Buitrago, E.; Fallica, R.; Fan, D., et al., "SnOx high-efficiency EUV interference lithography gratings towards the ultimate resolution in photolithography," Microelectronic Engineering(155), 44-49, (2016).

[14] Prezioso, S.; De Marco, P.; Zuppella, P., et al., "A study of the mechanical vibrations of a table-top extreme ultraviolet interference nanolithography tool," Review of Scientific Instruments(81) 4, 045110, (2010).

[15] Stowers, J.; Keszler, D. A., "High resolution, high sensitivity inorganic resists," Microelectronic Engineering(86), 730-733, (2009).

[16] Wang, L.; Terhalle, B.; Guzenko, V. A., et al., "Generation of High-resolution Kagome Lattice Structures Using Extreme Ultraviolet Interference Lithography," Applied Physics Letters(101) 9, 093104, (2012).

[17] Brink, M. v. d. "Cost-effective shrink with Holistic Lithography, extended by EUV," Proc. EUVL Symposium, Maastricht, The Neatherlands, (2015).

[18] Buitrago, E.; Nagahara, S.; Yildirim, O., et al., "Sensitivity enhancement of chemically amplified resists and performance study using extreme ultraviolet interference lithography," Journal of Micro/Nanolithography, MEMS, and MOEMS(15) 3, 033502033502, (2016).

[19] Karim, W.; Tschupp, S. A.; Oezaslan, M., et al., "High-Resolution and Large-Area Nanoparticle Arrays Using EUV Interference Lithography," Nanoscale(7) 16, 7386-7393, (2015).

[20] Karim, W.; Kleibert, A.; Hartfelder, U., et al., "Size-dependent redox behavior of iron observed by in-situ single nanoparticle spectro-microscopy on well-defined model systems," Scientific Reports(6), 18818, (2016).

[21] Fan, D.; Buitrago, E.; Yang, S., et al., "Patterning of nanodot-arrays using EUV achromatic Talbot lithography at the Swiss Light Source and Shanghai Synchrotron Radiation Facility," Microelectronic Engineering(155), 55-60, (2016).

[22] Fallica, R.; Stowers, J. K.; Vockenhuber, M., et al. "Absorption Coefficient and Dill Parameters of CAR and non-CAR Resists at EUV," Proc. International Symposium on Extreme Ultraviolet Lithography, Maastricht, The Netherlands, pp P-RE-06, (2015). 
[23] Fallica, R.; Stowers, J. K.; Grenville, A., et al. "Dynamic absorption coefficients of CAR and non-CAR resists at EUV," Proc. pp 977612-977612-10, (2016).

[24] Biafore, J. J.; Smith, M. D.; Mack, C. A., et al. "Statistical Simulation of Photoresists at EUV and ArF," Proc. SPIE Advanced Lithography, San Jose, pp 727343-727343-10, (2009).

[25] Thackeray, J. W., "Materials challenges for sub-20-nm lithography," Journal of Micro/Nanolithography, MEMS, and MOEMS(10) 3, 033009-033009-8, (2011). 